\title{
SOME TRIGONOMETRIC IDENTITIES RELATED TO EXACT COVERS
}

\author{
JOHN BEEBEE
}

(Communicated by William Adams)

\begin{abstract}
Sherman K. Stein proves that if $\sin \pi z=k \prod_{i=1}^{n} \sin \left(\pi / d_{i}\right)\left(b_{i}-z\right)$ where the $b_{i}$ are integers, the $d_{i}$ are positive integers, $k$ is a constant, then $\left\{\left(d_{i}: b_{i}\right)\right\}$ is an exact cover. It is shown here that if $0 \leq b_{i}<d_{i}$ then $k=$ $-2^{n-1}$, that the converse is also true, and an analogous formula is conjectured for infinite exact covers. Many well known and lesser known trigonometric and functional identities can be derived from this result and known families of exact covers. A procedure is given for constructing exact covers by induction.
\end{abstract}

\section{PRELIMINARIES}

Let $(d: b)$ be the arithmetic progression (AP) $\{x: x=b+\alpha d, \alpha \in Z\}$. A finite collection of disjoint AP's $\left\{\left(d_{i}: b_{i}\right): 1 \leq i \leq n\right\}$ is called an exact cover if each integer belongs to precisely one AP. The numbers $d_{i}$ are the moduli and the numbers $b_{i}$ are the offsets. Since $\left(d_{i}: b_{i}\right)=\left(d_{i}: b_{i}+\gamma d_{i}\right)$ it is usual to assume that the offsets have been standardized, $0 \leq b_{i}<d_{i}$. A necessary and sufficient condition for a finite collection of AP's to be an exact cover is that $\sum_{i=1}^{n} 1 / d_{i}=1$ and $\left(d_{i}, d_{j}\right) \backslash b_{i}-b_{j}$. As a consequence there is only one AP in an exact cover with $b_{i}=0\left(\bmod d_{i}\right)$. If we let $n=\infty$ the collection of AP's is called an infinite exact cover. For an infinite exact cover it is possible for either $\sum_{i=1}^{\infty} 1 / d_{i}<1$, in which case the cover is called unsaturated, or $\sum_{i=1}^{\infty} 1 / d_{i}=1$, in which case it is saturated.

In [7] (Corollary 6.5) Stein proves that if

$$
\sin \pi z=k \prod_{i=1}^{n} \frac{\pi}{d_{i}}\left(b_{i}-z\right)
$$

where the $b_{i}$ are integers, the $d_{i}$ are positive integers, and $k$ is a constant then $\left\{\left(d_{i}: b_{i}\right)\right\}$ is an exact cover. Trigonometric identities like (1), for example

(2) $\sin n \theta=2^{n-1} \sin \theta \sin \left(\theta+\frac{\pi}{n}\right) \sin \left(\theta+\frac{2 \pi}{n}\right) \cdots \sin \left(\theta+\frac{(n-1) \pi}{n}\right)$

Received by the editors January 18, 1990 and, in revised form, May 4, 1990.

1980 Mathematics Subject Classification (1985 Revision). Primary 11B25; Secondary 33A10, $68 \mathrm{~T} 15$.

Key words and phrases. Exact covering systems, functional identities, trigonometric identities. 
appear frequently in analysis books. (See Hobson [3, p. 119]). Many other identities can be derived from (1) or (2) by taking derivatives of both sides, dividing both sides by $\sin \pi z / d_{1}$ and taking $\lim (z \rightarrow 0)$, choosing special values for $z$, etc. (See Hansen [2], particularly formulas 24, 29, and 91.) I show here that if $\left\{\left(d_{i}: b_{i}\right): 1 \leq i \leq n\right\}$ is an exact cover with standardized offsets then (1) is true with $k=-2^{n-1}$, and derive an analogous formula that I conjecture is true for the case $n=\infty$.

\section{CONSTRUCTION OF EXACT COVERS}

I first show how to construct exact covers by induction on the number of AP's in the cover. There is an exact parallel between the method of construction for exact covers given here and the trigonometric proof of an identity like (1). This parallel could be used to write a computer program to create a proof of any such identity. Theorem 1 below says that to verify an identity like (1) it is only necessary to show that $\left\{\left(d_{i}: b_{i}\right): 1 \leq i \leq n\right\}$ is an exact cover.

\section{The ReDuction of $C \bmod (q: k)$}

Let $\mathrm{C}$ be an exact cover, and let $(q: k)$ be an AP. Every number in $(q: k)$ belongs to exactly one AP in $C$, so for each integer $\alpha$ there is a unique index $i$ and integer $\gamma$ such that

$$
k+\alpha q=b_{i}+\gamma d_{i},
$$

where we must have $\left(d_{i}, q\right) \mid b_{i}-k$. For each $i$ with $\left(d_{i}, q\right) \mid b_{i}-k$ there is a unique particular solution, $\alpha=a_{i}$ to (3) with

$$
0 \leq a_{i}<d_{i} /\left(d_{i}, q\right) \text { and } a_{i} q /\left(d_{i}, q\right)=\left(b_{i}-k\right) /\left(d_{i}, q\right)+\gamma_{i} d_{i} /\left(d_{i}, q\right)
$$

and $\alpha$ satisfies $(3)$ if and only if $\alpha=a_{i}\left(\bmod d_{i} /\left(d_{i}, q\right)\right)$; i.e. $\alpha \in\left(d_{i} /\left(d_{i}, q\right)\right.$ : $\left.a_{i}\right)$. But every integer $\alpha$ satisfies (3) for exactly one $i$ with $\left(d_{i}, q\right) \mid b_{i}-k$. This proves that $\left\{\left(d_{i} /\left(d_{i}, q\right): a_{i}\right):\left(d_{i}, q\right) \mid b_{i}-k\right\}$ is an exact cover, called the reduction of $C \bmod (q: k)$. (See Simpson [5].)

Now let $q$ be prime, let $0 \leq k<q$, and let $B_{k}$ be the reduction of $C \bmod$ $(q: k)$. To indicate that the offset $a_{i}$ exists for a particular combination of $i$ and $k$ I will write $a_{i}=a_{i}(k)$. Then

(5)

$$
\begin{aligned}
B_{k} & =\left\{\left(d_{i}: a_{i}(k)\right):\left(d_{i}, q\right)=1\right\} \cup\left\{\left(d_{i} / q: a_{i}(k)\right):\left(d_{i}, q\right)=q \text { and } q \mid b_{i}-k\right\} \\
& =A_{k} \cup A_{k}^{\prime}
\end{aligned}
$$

is an exact cover. If $\left(d_{i}, q\right)=q$ for some $i, B_{k}$ must have fewer AP's than $C$, because the sum of the reciprocals of its moduli must be 1 .

Example 1. Let $C=\{(6: 0),(6: 2),(10: 1),(10: 5),(10: 7),(10: 9)$, $(15: 13),(30: 3),(30: 4),(30: 10),(30: 16),(30: 22),(30: 23)\}=\left\{\left(d_{i}:\right.\right.$ $\left.\left.b_{i}\right): 1 \leq i \leq 13\right\}$. Let $q=2$. Then $B_{0}$ and $B_{1}$ are calculated from (5) and 
the offsets $a_{i}(0)$ and $a_{i}(1)$ are calculated from (4).

$$
\begin{aligned}
& B_{0}=A_{0} \cup A_{0}^{\prime} \\
& =\{(15: 14)\} \cup\{(3: 0),(3: 1),(15: 2),(15: 5),(15: 8),(15: 11)\} \\
& =\left\{\left(d_{7}: a_{7}(0)\right)\right\} \cup\left\{\left(d_{1} / 2: a_{1}(0)\right),\left(d_{2} / 2: a_{2}(0)\right),\left(d_{9} / 2: a_{9}(0)\right)\right. \text {, } \\
& \left.\left(d_{10} / 2: a_{10}(0)\right),\left(d_{11} / 2: a_{11}(0)\right),\left(d_{12} / 2: a_{12}(0)\right)\right\} \text {. } \\
& B_{1}=A_{1} \cup A_{1}^{\prime} \\
& =\{(15: 6)\} \cup\{(5: 0),(5: 2),(5: 3),(5: 4),(15: 1),(15: 11)\} \\
& =\left\{\left(d_{7}: a_{7}(1)\right)\right\} \cup\left\{\left(d_{3} / 2: a_{3}(1)\right),\left(d_{4} / 2: a_{4}(1)\right),\left(d_{5} / 2: a_{5}(1)\right)\right. \text {, } \\
& \left.\left(d_{6} / 2: a_{6}(1)\right),\left(d_{8} / 2: a_{8}(1)\right),\left(d_{13} / 2: a_{13}(1)\right)\right\} \text {. }
\end{aligned}
$$

Lemma 1. If $\left(d_{i}, q\right)=1$ then $a_{i}(k)$ exists for all $k$ so $\left(d_{i}: a_{i}(k)\right) \in A_{k}$ for all $k$ and $\bigcup_{k=0}^{q-1}\left(d_{i} q: k+a_{i}(k) q\right)=\left(d_{i}: b_{i}\right)$.

Proof. If $\left(d_{i}, q\right)=1$ there is a solution $a_{i}(k)$ and $\gamma_{i}(k)$ to (4) for every $k$. By $(4)$, as $k$ varies over its range, $\gamma_{i}(k)(\bmod q)$ takes on each integer value in the same range. Thus $\bigcup_{k=0}^{q-1}\left(d_{i} q: k+a_{i}(k) q\right)=\bigcup_{k=0}^{q-1}\left(d_{i} q: b_{i}+\gamma_{i}(k) d_{i}\right)=$ $\left(d_{i}: b_{i}\right)$.

Example 2. In example $1\left(d_{7}, q\right)=(15,2)=1$ so $(15: 14)=\left(d_{7}: a_{7}(0)\right) \in A_{0}$ and $(15: 6)=\left(d_{7}: a_{7}(1)\right) \in A_{1}$ and $\left(d_{7} \cdot 2: 0+a_{7}(0) \cdot 2\right) \cup\left(d_{7} \cdot 2: 1+a_{7}(1) \cdot 2\right)=$ $(30: 28) \cup(30: 13)=(15: 13)=\left(d_{7}: b_{7}\right)$.

Lemma 2. If $q \mid d_{i}$ there is exactly one value of $k, 0 \leq k<q$, for which $a_{i}(k)$ exists, so $\left(d_{i} / q: a_{i}(k)\right) \in A_{k}^{\prime}$ for this value of $k$ and $\left(d_{i}: k+a_{i}(k) q\right)=\left(d_{i}: b_{i}\right)$.

Proof. Clearly $q \mid b_{i}-k$ for some value of $k$, so $a_{i}(k)$ exists for this value of $k$. If $a_{i}(r)$ exists, $0 \leq r<q$, then $q \mid b_{i}-r$ and $q \mid b_{i}-k$, so $q \mid k-r$. This implies $k=r$, so $k$ is unique. If $q \mid d_{i},\left(d_{i}: b_{i}\right)$ must be a subset of some $\operatorname{AP}(q: k), 0 \leq k<q$. By (3), $k+a_{i}(k) q=b_{i}+\gamma d_{i}$. Thus $\left(d_{i}: k+a_{i}(k) q\right)=\left(d_{i}: b_{i}\right)$.

Example 3. In example 1, $2 \mid d_{i}$ for $i \neq 7$. Thus $a_{i}(0)$ exists for $i=$ $1,2,9,10,11,12$ and $a_{i}(1)$ exists for $i=3,4,5,6,8,13$. For instance, if $k=1$ and $i=8$ then $\left(d_{8}: 1+a_{8}(1) \cdot 2\right)=(30: 1+1 \cdot 2)=(30: 3)=$ $\left(d_{8}: b_{8}\right)$. The generalization of the partition $\{7\} \cup\{1,2,9,10,11,12\} \cup$ $\{3,4,5,6,8,13\}$ to arbitrary primes $q$ and exact covers $C$ has appeared previously in the literature about Znám's conjecture (see Simpson [6] and Znám [8]).

The following lemma describes a class of exact covers called the natural irreducible covers by Korec [4], who also describes constructions 1 and 2 below.

Lemma 3. If $q$ is a prime, then $Z_{q}=\{(q: k): 0 \leq k<q\}$ is an exact cover. Also, $Z_{1}=\{(1: 0)\}$ is an exact cover. 
Construction 1. If $C_{1}=\left\{\left(d_{i}: b_{i}\right): 1 \leq i \leq n\right\}$ and if $C_{2}=\left\{\left(e_{j}: c_{j}\right): 1 \leq j \leq\right.$ $m\}$ are exact covers, then $C_{3}=\left\{\left(d_{i}: b_{i}\right): i \neq I\right\} \cup\left\{\left(e_{j} d_{I}: b_{I}+c_{j} d_{I}\right): 1 \leq j \leq m\right\}$ is an exact cover with $n+m-1$ AP's.

Korec writes $C_{3}=\operatorname{Split}\left(C_{1}, b_{I}, C_{2}\right)$. I call this construction covering $\left(d_{I}\right.$ : $b_{I}$ ) with $C_{2}$.

Example 4. Let $Z_{2}=\{(2: 0),(2: 1)\}$. Cover $(2: 0)$ with $B_{0}$ from Example 1 , getting the exact cover $\{(30: 28),(6: 0),(6: 2),(30: 4),(30: 10),(30:$ $16),(30: 22),(2: 1)\}$. Cover $(2: 1)$ in the latter with $B_{1}$, getting

$$
\begin{aligned}
C^{\prime}=\{(30: 28),(6: 0),(6: 2),(30: 4),(30: 10),(30: 16),(30: 22), \\
(30: 13),(10: 1),(10: 5),(10: 7),(10: 9),(30: 3),(30: 23)\} .
\end{aligned}
$$

Construction 2. Let $C_{1}=\left\{\left(d_{i}: b_{i}\right): 1 \leq i \leq n\right\}$ be an exact cover. Suppose the union of some subcollection $D=\left\{\left(d_{i_{j}}: b_{i_{j}}\right): 1 \leq j \leq m\right\}$ is the AP $(d: b)$. Then $C_{3}=\left(C_{1}-D\right) \cup\{(d: b)\}$ is an exact cover with $n-m+1$ AP's.

I call this construction the substitution of $(d: b)$ for $D$. Note that $d \mid d_{i_{j}}$ and $d \mid b_{i_{j}}-b$, so $C_{2}=\left\{\left(d_{i_{j}} / d:\left(b_{i_{j}}-b\right) / d: 1 \leq j \leq m\right\}\right.$ is an exact cover, the reduction of $C_{1} \bmod (d: b)$. If we cover $(d: b)$ with $C_{2}$, we just get $C_{1}$ back again, so Constructions 1 and 2 are inverses to each other.

Example 5. Let $D=\{(30: 28),(30: 13)\}$. From Example $2(30: 28) \cup$ $(30: 13)=(15: 13)$, so substitute $(15: 13)$ for $D$ in $C^{\prime}$ from Example 4 getting

$$
\begin{array}{r}
C^{\prime \prime}=\{(15: 13),(6: 0),(6: 2),(30: 4),(30: 10),(30: 16),(30: 22), \\
(10: 1),(10: 5),(10: 7),(10: 9),(30: 3),(30: 23)\} .
\end{array}
$$

Except for the order of the AP's $C^{\prime \prime}$ is the same as $C$ from Example 1. The intuitive meaning of the following lemma and its proof is that any exact cover $C$ can be disassembled and reassembled in the same way. I will choose $q$ so that the covers $B_{k}$ always have fewer AP's than $C$. Thus it gives a theoretical method to construct an exact cover by induction. Here it is used to prove Theorem 1 below, which shows that there is an exact parallel between the proof of identities like (1) and the construction of exact covers.

Lemma 4. Suppose we have a list of all exact covers with $M$ or fewer AP's. Any exact cover $C$ with $M+1$ AP's, except $Z_{M+1}$ (if $M+1$ is prime) can be constructed by first using Construction 1 to cover the AP's $(q: k) \in Z_{q}$ with $q$ exact covers from the list, where $q$ is a prime that is a proper divisor of some modulus of $C$. This gives an exact cover $C^{\prime}$. If $q$ does not divide every modulus of $C$, it will then be necessary to apply Construction 2 to some subcollections of $C^{\prime}$ to get $C$.

Proof. Let $C=\left\{\left(d_{i}: b_{i}\right): 1 \leq i \leq M+1\right\}$ be an exact cover, where $C$ is not the natural irreducible cover $Z_{M+1}$ described in Lemma 3 . Then there is a 
prime $q$ which is a proper divisor of at least one modulus of $C$. Let $B_{k}$ be the reduction of $C \bmod (q: k), 0 \leq k<q$. By the remark following (5) the $B_{k}$ 's have fewer AP's than $C$ and hence are in the list of exact covers with $M$ or fewer AP's. The proof of the lemma will be completed by showing how to reconstruct $C$ from the $B_{k}$ 's and $Z_{q}$. The procedure is illustrated in Examples 4 and 5 above and Example 6 below.

For each $k$ cover the AP $(q: k) \in Z_{q}$ with $B_{k}$. From (5) and Construction 1 we obtain the exact cover

$$
\begin{aligned}
C^{\prime}= & \left(\bigcup_{k=0}^{q-1}\left\{\left(d_{i} q: k+a_{i}(k) q\right):\left(d_{i}, q\right)=1\right\}\right) \\
& \cup\left(\bigcup_{k=0}^{q-1}\left\{\left(d_{i}: k+a_{i}(k) q\right):\left(d_{i}, q\right)=q \text { and } q \mid b_{i}-k\right\}\right) .
\end{aligned}
$$

By Lemma $1, \bigcup_{k=0}^{q-1}\left(d_{i} q: k+a_{i}(k) q\right)=\left(d_{i}: b_{i}\right)$ for each $i$ with $\left(d_{i}, q\right)=1$. Using Construction 2, substitute $\left(d_{i}: b_{i}\right)$ for $D=\left\{\left(d_{i} q: k+a_{i}(k) q\right): 0 \leq k<\right.$ $q\}$, getting the new exact cover

$$
C^{\prime \prime}=\left\{\left(d_{i}: b_{i}\right):\left(d_{i}, q\right)=1\right\} \cup \bigcup_{k=0}^{q-1}\left\{\left(d_{i}: k+a_{i}(k) q\right):\left(d_{i}, q\right)=q \text { and } q \mid b_{i}-k\right\} .
$$

By Lemma 2 if $\left(d_{i}, q\right)=q$ then $\left(d_{i}: k+a_{i}(k) q\right)=\left(d_{i}: b_{i}\right)$, so

$$
C^{\prime \prime}=\left\{\left(d_{i}: b_{i}\right):\left(d_{i}, q\right)=1\right\} \cup\left\{\left(d_{i}: b_{i}\right):\left(d_{i}, q\right)=q\right\}=C .
$$

In the preceding proof all of the AP's except perhaps those belonging to $C$ have standardized offsets. Thus, when Constructions 1 and 2 are used to build an exact cover with standardized offsets we can assume that we are applying Constructions 1 and 2 to AP's with standardized offsets.

Example 6. Let $C$ be as in Example 1, but let $q=5$. Then

$$
\begin{aligned}
& B_{0}=A_{0} \cup A_{0}^{\prime}=\{(6: 0),(6: 4)\} \cup\{(2: 1),(6: 2)\} \\
& B_{1}=A_{1} \cup A_{1}^{\prime}=\{(6: 1),(6: 5)\} \cup\{(2: 0),(6: 3)\} \\
& B_{2}=A_{2} \cup A_{2}^{\prime}=\{(6: 2),(6: 0)\} \cup\{(2: 1),(6: 4)\} \\
& B_{3}=A_{3} \cup A_{3}^{\prime}=\{(6: 3),(6: 1)\} \cup\{(3: 2),(6: 0),(6: 4)\} \\
& B_{4}=A_{4} \cup A_{4}^{\prime}=\{(6: 4),(6: 2)\} \cup\{(2: 1),(6: 0)\}
\end{aligned}
$$

Cover the AP $(5: k) \in Z_{5}$ with $B_{k}, 0 \leq k<5$ getting

$$
\begin{aligned}
C^{\prime}= & (\{(30: 0),(30: 20)\} \cup\{(30: 6),(30: 26)\} \cup\{(30: 12),(30: 2)\} \\
& \cup\{(30: 18),(30: 8)\} \cup\{(30: 24),(30: 14)\}) \\
& \bigcup(\{(10: 5),(30: 10)\} \cup\{(10: 1),(30: 16)\} \cup\{(10: 7),(30: 22)\} \\
& \cup\{(15: 13),(30: 3),(30: 23)\} \cup\{(10: 9),(30: 4)\}) .
\end{aligned}
$$


Now $(30: 0) \cup(30: 6) \cup(30: 12) \cup(30: 18) \cup(30: 24)=(6: 0)$ and $(30: 20) \cup(30: 26) \cup(30: 2) \cup(30: 8) \cup(30: 14)=(6: 2)$. Making these substitutions in $C^{\prime}$ we get

$$
\begin{aligned}
C^{\prime \prime} & =\{(6: 0),(6: 2)\} \cup\{(10: 5),(30: 10),(10: 1),(30: 16),(10: 7), \\
& (30: 22),(15: 13),(30: 3),(30: 23),(10: 9),(30: 4)\} \\
& =C .
\end{aligned}
$$

\section{TRIGONOMETRIC IDENTITIES}

Theorem 1. The set of AP's $C=\left\{\left(d_{i}: b_{i}\right): 1 \leq i \leq n\right\}$, with standardized offsets, is an exact cover if and only if

$$
\sin \pi z=-2^{n-1} \prod_{i=1}^{n} \sin \frac{\pi}{d_{i}}\left(b_{i}-z\right) .
$$

If the offsets are not standardized then write $b_{i}=b_{i}^{\prime}+\eta_{i} d_{i}$, where $0 \leq b_{i}^{\prime}<d_{i}$. The product on the right in (6) must then be multiplied by $(-1)^{\sum_{i=1}^{n} \eta_{i}}$.

Proof. If (6) holds then $C$ is an exact cover because the zeros of the function on the left must be the same as the zeros of the function on the right and have the same multiplicity.

The first proof of the converse uses the inductive construction of exact covers described in Lemma 4. A different proof is given after Corollary 1 below. Substitute $-\pi z=n \theta$ in (2), getting the identity

$$
\sin \pi z=-2^{n-1} \prod_{k=0}^{n-1} \frac{\pi}{n}(k-z) .
$$

Thus Theorem 1 is true for the covers $Z_{q}$ described in Lemma 3.

To complete the proof it is necessary to show that if covers $C_{1}$ and $C_{2}$ satisfy (6), then the cover $C_{3}$ obtained from Construction 1 or 2 satisfies (6). If $C=\left\{\left(d_{i}: b_{i}\right): 1 \leq i \leq n\right\}$ let

$$
\prod C=-2^{n-1} \prod_{i=1}^{n} \sin \frac{\pi}{d_{i}}\left(b_{i}-z\right) .
$$

Consider Construction 1 and suppose

$$
\begin{aligned}
\sin \pi z & =-2^{n-1} \prod_{i=1}^{n} \sin \frac{\pi}{d_{i}}\left(b_{i}-z\right) \\
& =-2^{m-1} \prod_{j=1}^{m} \sin \frac{\pi}{e_{j}}\left(c_{j}-z\right) .
\end{aligned}
$$


Then

$$
\begin{aligned}
\sin \frac{\pi}{d_{I}}\left(z-b_{I}\right) & =-2^{m-1} \prod_{j=1}^{m} \sin \frac{\pi}{e_{j}}\left(c_{j}-\frac{z-b_{I}}{d_{I}}\right) \\
& =-2^{m-1} \prod_{j=1}^{m} \sin \frac{\pi}{e_{j} d_{I}}\left(b_{I}+c_{j} d_{I}-z\right) .
\end{aligned}
$$

Substituting the latter in (7),

$$
\sin \pi z=-2^{m+n-2} \prod_{\substack{i=1 \\ i \neq I}}^{n} \sin \frac{\pi}{d_{i}}\left(b_{i}-z\right) \prod_{j=1}^{m} \sin \frac{\pi}{e_{j} d_{I}}\left(b_{I}+c_{j} d_{I}-z\right)=\prod C_{3} .
$$

Now consider Construction 2. Since $\bigcup_{j=1}^{m}\left(d_{i_{j}}: b_{i_{j}}\right)=(d: b)$ we can show that $d \mid d_{i_{j}}$ and $d \mid b_{i_{j}}-b$ and $\left\{\left(d_{i_{j}} / d:\left(b_{i_{j}}-b\right) / d\right): 1 \leq j \leq m\right\}$ is an exact cover, the reduction of $C_{1} \bmod (d: b)$. Thus

$$
\begin{aligned}
\sin \pi z & =-2^{m-1} \prod_{j=1}^{m} \sin \frac{\pi}{d_{i_{j}} / d}\left(\left(b_{i_{j}}-b\right) / d-z\right) \\
& =-2^{m-1} \prod_{j=1}^{m} \sin \frac{\pi}{d_{i_{j}}}\left(b_{i_{j}}-b-d z\right) .
\end{aligned}
$$

Substituting $(z-b) / d$ for $z$ in the last equation,

$$
\sin \frac{\pi}{d}(z-b)=-2^{m-1} \prod_{j=1}^{m} \sin \frac{\pi}{d_{i_{j}}}\left(b_{i_{j}}-z\right)
$$

Substituting for $\prod_{j=1}^{m} \sin \left(\pi / d_{i_{j}}\right)\left(b_{i_{j}}-z\right)$ in $(7)$,

$$
\sin \pi z=-2^{n-m} \sin \frac{\pi}{d}(b-z) \prod_{\substack{i=1 \\ i \neq i_{j}}}^{n} \sin \frac{\pi}{d_{i}}\left(b_{i}-z\right)=\prod C_{3} .
$$

If the offsets are not standardized, let $b_{i}=b_{i}^{\prime}+\eta_{i} d_{i}$, where $0 \leq b_{i}^{\prime}<d_{i}$. Then

$$
\sin \frac{\pi}{d_{i}}\left(b_{i}-z\right)=(-1)^{\eta_{i}} \sin \frac{\pi}{d_{i}}\left(b_{i}^{\prime}-z\right),
$$

so

$$
\sin \pi z=(-1)^{\sum_{i=1}^{n} \eta_{i}}\left(-2^{n-1}\right) \prod_{i=1}^{n} \sin \frac{\pi}{d_{i}}\left(b_{i}-z\right)
$$

Corollary 1. Suppose $\left(d_{1}: b_{1}\right)$ is the unique AP in the exact cover $\left\{\left(d_{i}: b_{i}\right)\right.$ : $1 \leq i \leq n\}$ with $b_{1}=0$ and the offsets are standardized. Then

$$
\frac{d_{1}}{2^{n-1}}=\prod_{i=2}^{n} \sin \frac{\pi b_{i}}{d_{i}}
$$


and hence

$$
\sin \pi z=d_{1} \sin \frac{\pi z}{d_{1}} \prod_{i=2}^{n} \frac{\sin \left(\pi / d_{i}\right)\left(b_{i}-z\right)}{\sin \left(\pi b_{i} / d_{i}\right)}
$$

Proof. Divide both sides of $(6)$ by $\sin \left(\pi / d_{1}\right)\left(b_{1}-z\right)$ and take $\lim (z \rightarrow 0)$ on both sides.

I now give a proof of Corollary 1 (and hence Theorem 1 ) that is quite different from the one just given. This proof should make the conjecture below more plausible.

Proof of Corollary 1. For each real number $\rho>0$ define

$$
f_{\rho}(z)=\pi z \prod_{k=1}^{\rho}\left(1-\frac{z}{k}\right)\left(1+\frac{z}{k}\right)=\pi z \prod_{\substack{k=-\rho \\ k \neq 0}}^{\rho}\left(1-\frac{z}{k}\right),
$$

where the notation $\prod_{k=-\rho}^{\rho}$ means that the product is to be evaluated for integers $k,-\rho \leq k \leq \rho$. Now let $C=\left\{\left(d_{i}: b_{i}\right): 1 \leq i \leq n\right\}$ be an exact cover with $b_{1}=0$. Then

$$
f_{\rho}(z)=\pi z \prod_{\substack{k=-\rho / d_{1} \\ k \neq 0}}^{\rho / d_{1}}\left(1-\frac{z}{k d_{1}}\right) \prod_{i=2}^{n}\left(\prod_{k=\left(-\rho-b_{i}\right) / d_{i}}^{\left(\rho-b_{i}\right) / d_{i}}\left(1-\frac{z}{b_{i}+k d_{i}}\right)\right)
$$

From Hobson $\left[3\right.$, p. 350] we know that $\lim _{\rho \rightarrow \infty} f_{\rho}(z)=\sin \pi z$.

But if $b_{i} \neq 0$ then

$$
\begin{aligned}
f_{\rho}\left(\frac{b_{i}-z}{d_{i}}\right) & =\pi\left(\frac{b_{i}-z}{d_{i}}\right) \prod_{\substack{k=-\rho \\
k \neq 0}}^{\rho}\left(1-\frac{b_{i}-z}{k d_{i}}\right) \\
& =\pi \frac{b_{i}}{d_{i}}\left(1-\frac{z}{b_{i}}\right) \prod_{\substack{k=-\rho \\
k \neq 0}}^{\rho}\left(\frac{k d_{i}-b_{i}}{k d_{i}}+\frac{z}{k d_{i}}\right) \\
& =\pi \frac{b_{i}}{d_{i}}\left(1-\frac{z}{b_{i}}\right) \prod_{\substack{k=-\rho \\
k \neq 0}}^{\rho}\left(1-\frac{b_{i}}{k d_{i}}\right)\left(1+\frac{z}{k d_{i}-b_{i}}\right) \\
& =f_{\rho}\left(\frac{b_{i}}{d_{i}}\right) \prod_{k=-\rho}^{\rho}\left(1-\frac{z}{b_{i}+k d_{i}}\right) .
\end{aligned}
$$

Hence

$$
f_{\rho / d_{i}}\left(\frac{b_{i}-z}{d_{i}}\right)=f_{\rho / d_{i}}\left(\frac{b_{i}}{d_{i}}\right) \prod_{k=-\rho / d_{i}}^{\rho / d_{i}}\left(1-\frac{z}{b_{i}+k d_{i}}\right)
$$


Thus

$$
\begin{aligned}
\sin \pi\left(\frac{b_{i}-z}{d_{i}}\right) & =\lim _{\rho \rightarrow \infty} f_{\rho / d_{i}}\left(\frac{b_{i}-z}{d_{i}}\right) \\
& =\sin \frac{\pi b_{i}}{d_{i}}\left(\lim _{\rho \rightarrow \infty} \prod_{k=-\rho / d_{i}}^{\rho / d_{i}}\left(1-\frac{z}{b_{i}+k d_{i}}\right)\right) \\
& =\sin \frac{\pi b_{i}}{d_{i}}\left(\lim _{\rho \rightarrow \infty} \prod_{k=\left(-\rho-b_{i}\right) / d_{i}}^{\left(\rho-b_{i}\right) / d_{i}}\left(1-\frac{z}{b_{i}+k d_{i}}\right)\right) .
\end{aligned}
$$

Also

$$
\sin \frac{\pi z}{d_{1}}=\frac{\pi z}{d_{1}}\left(\lim _{\rho \rightarrow \infty} \prod_{\substack{k=-\rho / d_{1} \\ k \neq 0}}^{\rho / d_{1}}\left(1-\frac{z}{k d_{1}}\right)\right) .
$$

Taking the limit of both sides of (9) and substituting from (10) and (11) we get

$$
\sin \pi z=d_{1} \sin \frac{\pi z}{d_{1}} \prod_{i=2}^{n} \frac{\sin \left(\pi / d_{i}\right)\left(b_{i}-z\right)}{\sin \left(\pi b_{i} / d_{i}\right)} .
$$

If we could somehow let $n \rightarrow \infty$ as well, we could prove the conjecture below.

I will now show that if $b_{1}=0$, then $d_{1} / 2^{n-1}=\prod_{i=2}^{n} \sin \pi b_{i} / d_{i}$. Let $m=\operatorname{lcm}\left\{d_{1}, d_{2}, \ldots, d_{n}\right\}$. Since $C$ is an exact cover,

$$
x^{2 m}-1=\prod_{i=1}^{n}\left(x^{2 m / d_{i}}-e^{2 \pi i b_{i} / d_{i}}\right) .
$$

(See the proof of Theorem 5.1 in Stein [7].) Let $x=e^{\pi i z / m}$ in this equation. Then

$$
e^{2 \pi i z}-1=\prod_{i=1}^{n}\left(e^{2 \pi i z / d_{i}}-e^{2 \pi i b_{i} / d_{i}}\right) .
$$

Divide both sides by $e^{2 \pi i z / d_{1}}-e^{2 \pi i b_{1} / d_{1}}=e^{2 \pi i z / d_{1}}-1$, and take $\lim (z \rightarrow 0)$ of both sides, getting

$$
d_{1}=\prod_{i=2}^{n}\left(1-e^{2 \pi i b_{i} / d_{i}}\right)=\prod_{i=2}^{n} e^{\pi i b_{i} / d_{i}}\left(-2 i \sin \pi b_{i} / d_{i}\right)
$$

By Theorem 1 of Fraenkel [1] (with $\mathrm{n}=1$ ), if $0 \leq b_{i}<d_{i}$, then

$$
\sum_{i=1}^{n} \frac{b_{i}}{d_{i}}=\sum_{i=2}^{n} \frac{b_{i}}{d_{i}}=\frac{n-1}{2} \text {. }
$$

Thus

$$
d_{1}=2^{n-1} e^{\pi i(n-1) / 2} e^{-\pi i(n-1) / 2} \prod_{i=2}^{n} \sin \frac{\pi b_{i}}{d_{i}}=2^{n-1} \prod_{i=2}^{n} \sin \frac{\pi b_{i}}{d_{i}}
$$


Corollary 2. If $\left\{\left(d_{i}: b_{i}\right): 1 \leq i \leq n\right\}$ is an exact cover with standardized offsets then

(1) $-2^{n-1} \prod_{i=1}^{n} \sin \left(\pi / 2 d_{i}\right)\left(2 b_{i}-1\right)=1$,

(2) $\cot \pi z=\sum_{i=1}^{n}\left(1 / d_{i}\right) \cot \left(\pi / d_{i}\right)\left(z-b_{i}\right)$,

(3) $\csc ^{2} \pi z=\sum_{i=1}^{n}\left(1 / d_{i}^{2}\right) \csc ^{2}\left(\pi / d_{i}\right)\left(z-b_{i}\right)$.

Proof of (1). Let $z=1 / 2$ in (6).

Proof of (2). Take the logarithmic derivative of both sides of (6).

Proof of (3). Take the derivative of (2) of Corollary 2.

If $C=\left\{\left(d_{i}: b_{i}\right): 1 \leq i \leq n\right\}$ is an exact cover, and $q>0$ is relatively prime to all $d_{i}$ and $k$ is any integer, then $C^{\prime}=\left\{\left(d_{i}: k+q b_{i}\left(\bmod d_{i}\right)\right): 1 \leq i \leq n\right\}$ is an exact cover. More generally, if $d$ is a positive integer and $b$ is any integer, then $C^{\prime \prime}$, the reduction of $C \bmod (d: b)$ is an exact cover.

Corollary 3. If $\sin \pi z=\Pi C$, then $\sin \pi z=\Pi C^{\prime}=\Pi C^{\prime \prime}$.

Conjecture. If $\left\{\left(d_{i}: b_{i}\right): 1 \leq i<\infty\right\}$ is an infinite exact cover (saturated or unsaturated $)$ with $b_{1}=0\left(\bmod d_{1}\right)$ and standardized offsets then

$$
\sin \pi z=d_{1} \sin \frac{\pi z}{d_{1}} \prod_{i=2}^{\infty} \frac{\sin \left(\pi / d_{i}\right)\left(b_{i}-z\right)}{\sin \pi b_{i} / d_{i}} .
$$

\section{REFERENCES}

1. Aviezri S. Fraenkel, A characterization of exactly covering congruences, Discrete Math. 4 (1973), 359-366.

2. Eldon R. Hansen, A table of series and products, Prentice Hall, Englewood Cliffs, NJ, 1975.

3. E. W. Hobson, $A$ treatise on plane and advanced trigonometry, Dover, 1957.

4. Ivan Korec, Irreducible disjoint covering systems, Acta Arith. XLIV (1984), 389-395.

5. R. J. Simpson, Regular coverings of the integers by arithmetic progressions, Acta Arith. $\mathbf{4 5}$ (1985), 145-152.

6. __ Disjoint covering systems of congruences, Amer. Math. Monthly 94 (1987), 865-868.

7. Sherman K. Stein, Unions of arithmetic sequences, Math. Ann. 134 (1958), 289-294.

8. Š. Znám, A survey of covering systems of congruences, Acta Math. Univ. Comenian. 40-41 (1982), 59-79.

Department of Mathematical Sciences, University of Alaska at ANChorage, ANChorAGE, ALASKa 99508 